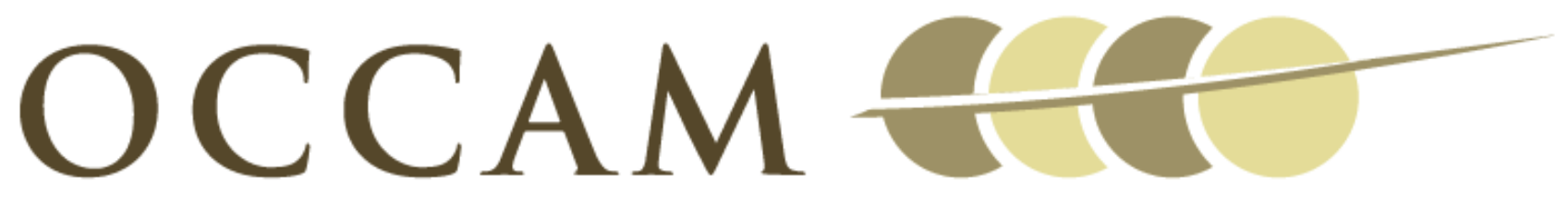

OXFORD CENTRE FOR COLLABORATIVE APPLIED MATHEMATICS

\author{
Report Number 10/37
}

Circumferential buckling instability of a growing cylindrical tube

$$
\text { by }
$$

D.E. Moulton and A. Goriely

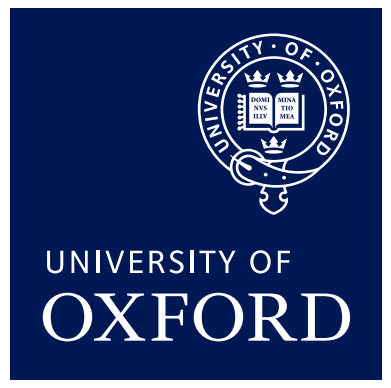

Oxford Centre for Collaborative Applied Mathematics Mathematical Institute 24 - 29 St Giles'

Oxford

OX1 3LB

England 



\title{
Circumferential buckling instability of a growing cylindrical tube
}

\author{
D.E. Moulton · A. Goriely
}

Received: date / Accepted: date

\begin{abstract}
A cylindrical elastic tube under uniform radial pressure will buckle circumferentially to a non-circular cross section at a critical pressure. The buckling represents an instability of the inner or outer edge of the tube. This is a common phenomenon in biological tissues, where it is referred to as mucosal folding. Here, we investigate this buckling instability in a growing elastic tube. A change in thickness due to growth can have a dramatic impact on circumferential buckling, both in the critical pressure and the buckling pattern. We consider both single and bi-layer tubes and multiple boundary conditions. We highlight the competition between geometric effects, i.e. the change in tube dimensions, and mechanical effects, i.e. the effect of residual stress, due to differential growth. This competition can lead to non-intuitive results, such as a tube growing to be thicker and yet buckle at a lower pressure.
\end{abstract}

Keywords elasticity $\cdot$ Buckling instability $\cdot$ Incremental deformation $\cdot$ growing tissue

\section{Introduction}

When an elastic tube is under radial pressure, a circumferential instability may develop at a critical pressure in which the tube buckles to a non-circular cross section. This instability is fundamentally different from the classical Euler's instability $[1,2]$ as it takes place in the cross-section of the tube rather than along its length. Collapsible elastic tubes which rely on this instability have been used in drug delivery pumps [3], where the buckling of a highly compliant tube and hence the rate of drug delivery is controlled by external pressure. Circumferential buckling is also a common phenomenon in a number of biological tissues, where it is referred to as mucosal folding. Mucosal folding is observed in the airways [4], esophagus [5,6], blood vessels [7], and gastrointestinal tract [8].

A number of models have been developed in the literature to study the mechanical and geometrical aspects of circumferential buckling [9-14,6]. One feature of interest is the effect of wall thickness on the buckling properties. This is of particular importance in airways:

Derek E. Moulton

OCCAM, Institute of Mathematics, University of Oxford, UK E-mail: moulton@maths.ox.ac.uk

Alain Goriely

OCCAM, Institute of Mathematics, University of Oxford, UK E-mail: goriely@maths.ox.ac.uk 
chronic asthma is characterized by structural changes to the airway walls, in which all layers of the airway wall become thicker $[15,16]$. These structural changes are referred to as airway remodelling [17], a complex phenomenon involving cellular, biochemical and mechanical responses.

A central question to many of the above mentioned studies is how thickness impacts circumferential buckling. What is the preferred wave instabilty? What is the critical pressure at which buckling occurs? It is important to realise that in biological structures a changes in thickness is the result of a growth process. Differential growth has been shown to have a dramatic impact on the mechanical response and stability of spherical shells [18] as well as cylindrical tubes under axial loading [19], but with circumferential buckling the element of growth is not present in previous studies. It is therefore potentially important to fully characterize the role and impact of growth in circumferential buckling of elastic tubes.

Differential growth refers to the anisotropic or inhomogeneous addition of mass, and is a common feature in biological tissues. Growth can play a key role in the stability of a biological structure, either through changes in geometry which enable the structure to better endure pressure, or by creating so-called residual stresses. Local changes of mass induce elastic stresses which cannot be eliminated by changes in geometry, and thus generate stresses which persist in the absence of external loads on the material - these are residual stresses. They are a hallmark of biological systems, and play a key role in the mechanical properties of numerous biological tissues, including blood vessels [20], arteries [21], and plant stems [22].

In a companion paper [23], we specialise the analysis of differential growth and buckling specifically to airways and show that differential growth may be a key component of airway remodelling. In this paper, we study the generic problem of circumferential instability in a two-layer tube with differential growth; our goal is to understand the role of external loads, tube geometry, and growth in the instability by identifying the critical buckling pressure and the buckling mode (i.e. number of folds in buckled state). We begin in Section 2 by formulating the model and the stability analysis, for hyperelastic, incompressible cylindrical materials. We formulate the buckling analysis using the incremental theory in finite elasticity. Growth of the tube is described through the theory of morphoelasticity and the decomposition of the deformation tensor [24]. In Section 3 we study a single layer tube, analyzing anisotropic growth as well as isotropic growth in a constrained geometry. The incremental equations are solved numerically and compared with an asymptotic analysis valid in the limit of thin tubes. In Section 4 we consider a bilayer tube, with each layer having different stiffness properties and different growth rates. We demonstrate that growth rates can be tuned to specify bifurcation properties.

\section{Background, Setup}

We consider the growth and deformation of an incompressible hyperelastic cylindrical tube. Our interest is in the circumferential buckling of a cross section, and so we restrict to deformations uniform along the tube axis. To formulate the stability analysis, we begin with the formulation of the initial radial deformation. We consider a tube of inner and outer radii $A$ and $B$. After deformation the function $r(R)$ describes the radius of a circle initially at radius $R$ with $r(A)=a$ and $r(B)=b$. The deformation gradient tensor is $\mathbf{F}=\operatorname{diag}\left(r^{\prime}(R), r / R, 1\right)$, expressed in cylindrical coordinates $(R, \Theta, Z)$ and $(r, \theta, z)$. Following previous work on morphoelastic materials [25-28], we decompose the deformation gradient into a growth part and elastic part $\mathbf{F}=\mathbf{A G}$. The elastic strain tensor is $\mathbf{A}=\operatorname{diag}\left(\alpha_{1}, \alpha_{2}, 1\right)$, where index 1 refers 
to the radial direction and index 2 the circumferential direction. Incompressibility implies $\operatorname{det} \mathbf{A}=1$ so we write $\mathbf{A}=\operatorname{diag}\left(\alpha^{-1}, \alpha, 1\right)$, where $\alpha:=\alpha_{2}$. The growth tensor for the symmetric growth is $\mathbf{G}=\operatorname{diag}\left(\gamma_{1}, \gamma_{2}, 1\right)$. Here $\gamma_{1}$ corresponds to radial growth, with each radial fiber gaining or losing mass if $\gamma_{1}$ is greater than or less than 1 , respectively, and $\gamma_{2}$ is circumferential growth. Isotropic growth occurs when $\gamma_{1}=\gamma_{2}$. If the $\gamma_{i}$ are functions of $R$, the growth is inhomogeneous. The decomposition $\mathbf{F}=\mathbf{A G}$ implies $r^{\prime}=\gamma_{1} / \alpha, r / R=\alpha \gamma_{2}$, from which the deformation is given by

$$
r^{2}-a^{2}=2 \int_{A}^{R} \gamma_{1} \gamma_{2} R d R
$$

Once the value of the inner radius $a$ is known, the deformation is completely determined. Let $t_{1}$ and $t_{2}$ be the radial and circumferential (hoop) stresses of the Cauchy stress tensor. In the absence of body forces, the equilibrium linear momentum equation is $\operatorname{div} \mathbf{T}=\mathbf{0}$, and the only non vanishing component is

$$
\frac{\partial t_{1}}{\partial r}+\frac{1}{r}\left(t_{1}-t_{2}\right)=0
$$

Hyperelasticity implies that there exists a strain energy function $W(\mathbf{A})$, from which the stress-strain constitutive relation is $\mathbf{T}=\mathbf{A} \frac{\partial W}{\partial \mathbf{A}}-p \mathbf{1}$, where $p$ is the hydrostatic pressure due to the incompressibility constraint. The components of the stress-strain relation are

$$
t_{1}=\alpha_{1} W_{1}-p, \quad t_{2}=\alpha_{2} W_{2}-p
$$

where $W_{i}=\frac{\partial W}{\partial \alpha_{i}}, t_{1}$ is the radial stress and $t_{2}$ the circumferential hoop stress. Eliminating $p$ in (3) and defining the auxiliary function $\hat{W}(\alpha)=W\left(\alpha^{-1}, \alpha\right)$, (2) gives the following closed equations for the stress:

$$
t_{1}(r)=\int_{a}^{r} \frac{\alpha \hat{W}^{\prime}(\alpha)}{r} d r, \quad t_{2}=t_{1}+\alpha \hat{W}^{\prime}(\alpha) .
$$

Defining $-P=t_{1}(b)-t_{1}(a)$, we have

$$
-P=\int_{a}^{b} \frac{\alpha \hat{W}^{\prime}(\alpha)}{r} d r=\int_{A}^{B} \frac{\gamma_{1} \hat{W}^{\prime}(\alpha)}{\gamma_{2} \alpha R} d R .
$$

Here $P$ is the applied load on the cylindrical tube, such that $P>0$ in the case of external pressure and $P<0$ for internal pressure. For given growth functions $\gamma_{i}$ and fixed pressure $P$, (5) is an equation for the unknown parameter $a$, since $b$ is related to $a$ through (1).

\subsection{Incremental deformation}

The approach to stability is to add a perturbation to the finite deformation (1). The perturbation comes in the form of an imposed incremental deformation from a wider class of deformation, i.e. without the symmetry of the finite deformation. Briefly, let $\chi^{(0)}$ be a known finite deformation and $\mathbf{T}^{(0)}$ the related Cauchy stress, also known. Introduce an incremental deformation $\chi^{(1)}$ and consider

$$
\chi=\chi^{(0)}+\varepsilon \chi^{(1)},
$$

where $\varepsilon$ is a small parameter characterizing the size of the imposed perturbation. The stability analysis proceeds by taking expansions of the deformation gradient and the elastic strain tensor (the growth tensor remains constant). That is, we write $\mathbf{F}=\operatorname{Grad} \chi=$ 
$\mathbf{F}^{(0)}+\varepsilon \mathbf{F}^{(1)} \mathbf{F}^{(0)}, \mathbf{A}=\mathbf{A}^{(0)}+\varepsilon \mathbf{A}^{(1)} \mathbf{A}^{(0)}$. The relation $\mathbf{F}=\mathbf{A} \mathbf{G}$ implies $\mathbf{F}^{(0)}=\mathbf{A}^{(0)} \mathbf{G}$ and $\mathbf{F}^{(1)}=\mathbf{A}^{(1)}$. Incompressibility implies $\operatorname{tr}\left(\mathbf{F}^{(1)}\right)=0$. We also expand the Cauchy stress tensor as $\mathbf{T}=\mathbf{T}^{(0)}+\varepsilon \mathbf{T}^{(1)}+O\left(\varepsilon^{2}\right)$ and the hydrostatic pressure as $p=p^{(0)}+\varepsilon p^{(1)}$. The stressstrain relation reads, to zeroth order,

$$
\mathbf{T}^{(0)}=\mathbf{A}^{(0)}\left(W_{\mathbf{A}}^{(0)}-p^{(0)} \mathbf{1}\right)
$$

and to first order

$$
\mathbf{T}^{(1)}=\mathbf{A}^{(1)}\left(\mathbf{T}^{(0)}+p^{(0)}\right)+\mathscr{L}: \mathbf{A}^{(1)}-p^{(1)} \mathbf{1},
$$

where $\mathscr{L}: \mathbf{A}^{(1)}=\mathbf{A}^{(0)} W_{\mathbf{A A}}^{(0)}: \mathbf{A}^{(1)} \mathbf{A}^{(0)}$. Here $W_{\mathbf{A}}^{(0)}, W_{\mathbf{A A}}^{(0)}$ are the first and second derivatives of $W$ with respect to $\mathbf{A}$, evaluated at $\mathbf{A}^{(0)}$. The components of the 4 th order tensor $\mathscr{L}$ are the instantaneous elastic moduli, these are functions of the strain components $\alpha_{i}$ and the derivatives of the strain energy $W\left(\alpha_{1}, \alpha_{2}\right)$ (see [29] for a derivation and formulas).

Setting $\operatorname{div} \mathbf{T}=\mathbf{0}$ gives at $O(1) \operatorname{div}\left(\mathbf{T}^{(0)}\right)=\mathbf{0}$, which is already satisfied by the finite deformation. At $O(\varepsilon)$, the incremental equilibrium equation may be written

$$
\mathbf{A}^{(1)} \operatorname{grad} p^{(0)}+\operatorname{div}\left(\mathscr{L}: \mathbf{A}^{(1)}\right)-\operatorname{grad} p^{(1)}=0 .
$$

Boundary conditions consist of prescribing either $\chi^{(1)}$ or $\mathbf{n} \cdot \mathbf{T}^{(1)}$, that is either the deformation or the traction are prescribed on the boundary.

For a given finite deformation, all terms at order zero are known. Then Equation (9) plus boundary conditions form the system for the linear stability analysis. If a solution to the boundary value problem exists, a bifurcation from the set of symmetric deformations is detected.

\subsection{Bifurcation analysis}

Since we only consider a cross section, the deformation is independent of the axial variable $z$, so we work in polar coordinates where the general form for the incremental deformation is $\chi^{(1)}=[u(r, \theta), v(r, \theta)]$. To first order, we have

$$
\mathbf{F}^{(1)}=\mathbf{A}^{(1)}=\left[\begin{array}{ll}
u_{r} & \frac{u_{\theta}-v}{r} \\
v_{r} & \frac{u+v_{\theta}}{r}
\end{array}\right] .
$$

The incompressibility condition is

$$
\operatorname{tr}\left(\mathbf{A}^{(1)}\right)=u_{r}+\left(u+v_{\theta}\right) / r=0 .
$$

Inserting (10) into the incremental equilibrium equation (9) yields two differential equations involving the unknown functions $u, v$, and $p^{(1)}$. To proceed, these functions are assumed to be of the form

$$
\begin{aligned}
& u(r, \theta)=f(r) \sin (n \theta) \\
& v(r, \theta)=g(r) \cos (n \theta) \\
& p^{(1)}(r, \theta)=h(r) \sin (n \theta) .
\end{aligned}
$$


The bifurcation is examined as a function of the buckling mode $n$, the number of folds in the buckled state. Making these substitutions and using the incompressibility condition (11) to solve for $g(r)$ in terms of $f(r)$, the system can be simplified to a single 4th order differential equation for $f(r)$. We consider in this paper a neo-Hookean tube characterized by strain energy function $W=\frac{\mu}{2}\left(\alpha_{1}^{2}+\alpha_{2}^{2}-2\right)$, where $\mu>0$ is the shear modulus. In this case, the governing equation is

$$
B_{4} f^{(4)}(r)+B_{3} f^{\prime \prime \prime}(r)+B_{2} f^{\prime \prime}(r)+B_{1} f^{\prime}(r)+B_{0} f(r)=0
$$

where

$$
\begin{aligned}
& B_{4}=\gamma_{2}^{2} R^{2}, \quad B_{3}=\frac{2 \gamma_{2}\left(\gamma_{1} \gamma_{2} R^{2}+2 r^{2}\right)}{r \gamma_{1}}, \\
& B_{2}=-\frac{3 \gamma_{2}{ }^{4} R^{4} \gamma_{1}-8 \gamma_{2}^{3} R^{2} r^{2}+n^{2} \gamma_{2}{ }^{4} R^{4} \gamma_{1}+r^{4} n^{2} \gamma_{1}}{r^{2} \gamma_{2}{ }^{2} R^{2} \gamma_{1}}, \\
& B_{1}=\frac{-3 r^{4} n^{2} \gamma_{1} \gamma_{2} R^{2}+2 r^{6} n^{2}+3 \gamma_{2}{ }^{5} R^{6} \gamma_{1}-4 \gamma_{2}{ }^{4} R^{4} r^{2}+n^{2} \gamma_{2}{ }^{5} R^{6} \gamma_{1}-2 \gamma_{2}{ }^{4} R^{4} n^{2} r^{2}}{r^{3} \gamma_{1} \gamma_{2}{ }^{3} R^{4}} \\
& B_{0}=\frac{-3 \gamma_{2}{ }^{4} R^{4} \gamma_{1}+4 \gamma_{2}{ }^{3} R^{2} r^{2}+3 n^{2} \gamma_{2}{ }^{4} R^{4} \gamma_{1}-4 \gamma_{2}{ }^{3} R^{2} n^{2} r^{2}-r^{4} n^{2} \gamma_{1}+r^{4} n^{4} \gamma_{1}}{r^{4} \gamma_{2} R^{2} \gamma_{1}}
\end{aligned}
$$

Note that $R=R(r)$ is determined from the finite deformation via (1). The system is closed with boundary conditions at $r=a$ and $b$. For example, zero shear and normal stress are expressed, respectively, as:

$$
\begin{aligned}
& \gamma_{1} \gamma_{2}^{4} R^{4} r^{3} f^{\prime \prime \prime}(r)+\left(2 \gamma_{2}^{3} R^{2} r^{4}+2 \gamma_{1} \gamma_{2}^{4} R^{4} r^{2}\right) f^{\prime \prime}(r)+\left(2 \gamma_{2}^{3} R^{2} r^{3}-2 \gamma_{1} \gamma_{2}^{4} n^{2} R^{4} r\right. \\
& \left.\quad-\gamma_{1} \gamma_{2}^{4} R^{4} r-\gamma_{1} n^{2} r^{5}\right) f^{\prime}(r)+\left(\gamma_{1} \gamma_{2}^{4} R^{4}-2 \gamma_{2}^{3} R^{2} r^{2}-\gamma_{1} \gamma_{2}^{4} R^{4}+2 \gamma_{2}^{3} n^{2} R^{2} r^{2}\right) f(r)=0 ; \\
& r^{2} f^{\prime \prime}(r)+r f^{\prime}(r)+\left(n^{2}-1\right) f(r)=0 .
\end{aligned}
$$

Alternatively, if part of the tube is held fixed at one boundary, the normal stress condition is replaced by the requirement that the perturbation vanish at that boundary. In this paper both types of conditions will be used.

With given boundary conditions, a solution to the boundary value problem (BVP) indicates the onset of instability. The bifurcation analysis proceeds by leaving one parameter in the system free and searching for a critical value of that parameter at which the BVP has a solution. This parameter might be the strain $\alpha$ at the inner boundary $r=a$, the applied pressure $P$, or the rate of growth. To demonstrate, suppose the external pressure $P$ is the bifurcation parameter. The system (13) with the chosen boundary conditions is solved numerically as follows. We write

$$
f(r)=a_{1} \xi_{1}(r)+a_{2} \xi_{2}(r) .
$$

Treating the system as an initial value problem at $r=a$, we solve two copies of the system with linearly independent initial conditions for the $\xi_{i}(r)$, such that both $\xi_{1}$ and $\xi_{2}$ satisfy the two boundary conditions at $r=a$. We integrate both $\xi_{i}$ forward to $r=b$ and form the determinant of boundary values at $r=b$

$$
D(P)=\left|\begin{array}{cc}
c_{1}\left(\xi_{1}(b) ; P\right) & c_{1}\left(\xi_{2}(b) ; P\right) \\
\left.c_{2}\left(\xi_{1}(b) ; P\right)\right) & c_{2}\left(\xi_{2}(b) ; P\right)
\end{array}\right|
$$


where $c_{1}(f(b))=0, c_{2}(f(b))=0$ are the boundary conditions at $r=b$. Due to linearity, if the determinant $D(P)=0$, then there exist values $a_{1}$ and $a_{2}$ for which $f(r)$ given by (15) solves the BVP. The parameter $P$ is iterated on until the determinant vanishes.

\subsection{Two layer model.}

The derivation and procedure outlined above is for a single layer tube. Extending the model to a tube of two connected layers with different material properties and growth rates in each layer is straightforward. Let the inner region be given in the initial configuration by $A \leq R \leq B$ and the outer region by $B \leq R \leq C$, and equivalently by $a \leq r \leq b$ and $b \leq r \leq c$ in the current configuration. Further, let $\mu^{(i)}, \gamma_{1}^{(i)}$, and $\gamma_{2}^{(i)}$ be the elastic modulus and growth functions in the inner region, and $\mu^{(o)}, \gamma_{1}^{(o)}$, and $\gamma_{2}^{(o)}$ the same in the outer region. The initial deformations for the inner and outer regions are given by

$$
r^{2}-a^{2}=\gamma_{1}^{(i)} \gamma_{2}^{(i)}\left(R^{2}-A^{2}\right), r^{2}-b^{2}=\gamma_{1}^{(o)} \gamma_{2}^{(o)}\left(R^{2}-B^{2}\right)
$$

Defining $P=t_{1}(a)-t_{1}(c)$ as the applied load, by continuity of radial stress at the interface $R=B$ we have

$$
-P=\int_{A}^{B} \frac{\gamma_{1}^{(i)}\left(\hat{W}^{(i)}\right)^{\prime}(\alpha)}{\gamma_{2}^{(i)} \alpha R} d R+\int_{B}^{C} \frac{\gamma_{1}^{(o)}\left(\hat{W}^{(o)}\right)^{\prime}(\alpha)}{\gamma_{2}^{(o)} \alpha R} d R .
$$

The stability analysis follows the same steps as the one layer model. Let $f_{1}(r), f_{2}(r)$ be the radial perturbations for the inner and outer regions, respectively. The boundary conditions zero tangential stress and either zero normal stress or zero perturbation are applied at $r=a$ and $r=c$. The 4 additional conditions needed at the interface $r=b$ are continuity of the perturbation,

$$
f_{1}=f_{2}, f_{1}^{\prime}=f_{2}^{\prime} \text { at } r=b
$$

and continuity of the stresses. Numerically, the approach is the same: we write $f_{1}=a_{1} \xi_{1}(r)+$ $a_{2} \xi_{2}(r), f_{2}=a_{1} \xi_{3}(r)+a_{2} \xi_{3}(r)$ and integrate two copies of the system forward from $r=a$. The end conditions at $r=b$ are used to determine initial conditions for $\xi_{3}$ and $\xi_{4}$, which are integrated to $r=c$. A determinant of boundary conditions at $r=c$ is formed, and bifurcation is detected when the determinant vanishes. The two layer model is analyzed in Section 4.

\section{One layer model}

The main issues we consider are how the buckling phenomenon is affected by imposed growth, both in the critical bifurcation values as well as the shape and mode of the buckled tube. First, it is useful to establish the case of no growth, that is the buckling of a tube under external pressure only. 


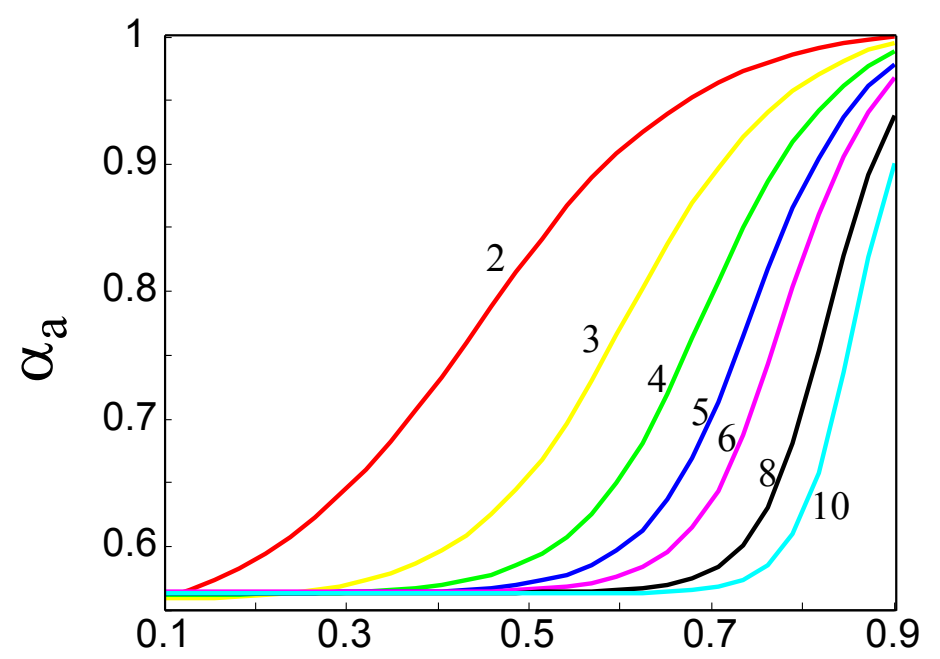

$\mathrm{A} / \mathrm{B}$

Fig. 1 Critical strain of a tube as a function of thickness, in the absence of growth.

\subsection{Buckling without growth}

We first consider incremental boundary conditions of zero normal and shear stress on both edges. As pressure is applied to the tube, the inner radius decreases until buckling occurs. Figure 1 shows the critical strain at the inner radius, $\alpha_{a}:=a / A$, plotted as a function of tube thickness for various modes. Since $\alpha_{a}=1$ for an unstrained tube, the critical strain for a fixed thickness is the first curve reached when moving down from $\alpha_{a}=1$, and the critical buckling mode is the corresponding mode. Over nearly the entire range of thicknesses, the critical mode is 2 , although a crossover to mode 6 occurs for very thick tubes $(A / B \approx 0.1$ and smaller). The shape of the curves is qualitatively similar to the buckling of a spherical shell under compression (see Fig. 9 in [18]), but with the marked difference that sequentially higher modes can be excited in the spherical shell case as $A / B$ increases, whereas most modes are not excitable in the cylindrical tube case presently under consideration.

\subsection{Effect of growth on stability}

We consider in this section homogeneous anisotropic growth. Again, the incremental boundary conditions are zero shear and normal stress at both $r=a$ and $r=b$. The growth functions $\gamma_{i}$ are constant, with the circumferential growth $\gamma_{2}=1$, and we vary the radial growth $\gamma_{1}$. In Figure 2, the bifurcation pressure $P_{c r}$ is plotted as a function of the radial growth $\gamma_{1}$, for various modes $n$ and for fixed tube thickness $A / B=0.5$. For a given $\gamma_{1}$, the smallest value of $P_{c r}$ over all modes is the critical external pressure, denoted $P_{c r}^{*}$, at which the tube becomes 


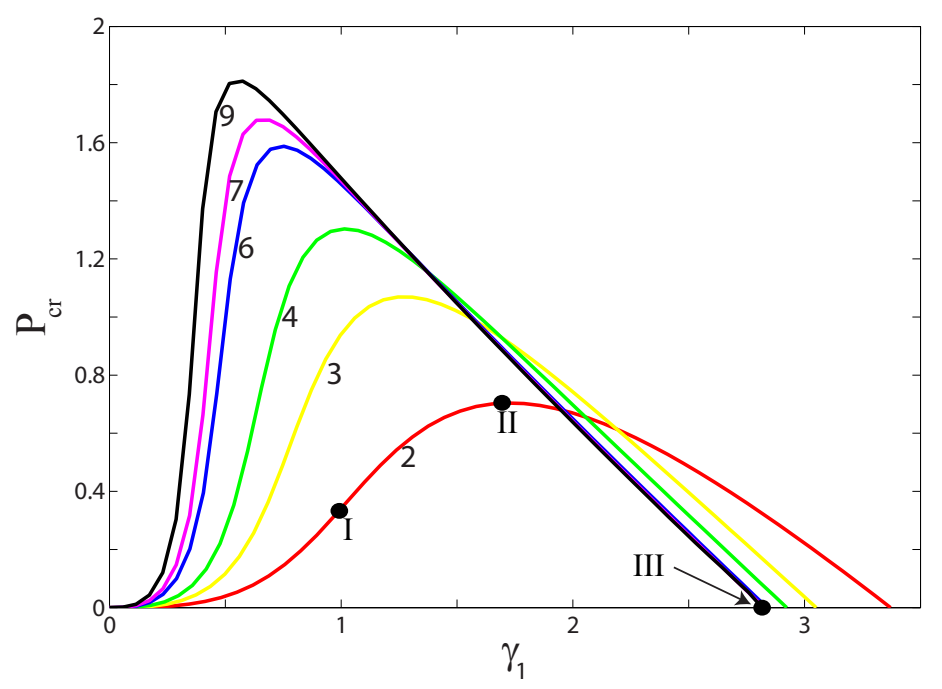

Fig. 2 Critical buckling pressure as a function of radial growth $\gamma_{1}$ for modes $n=2,3,4,6,7,9$. Other modes are not included because they do not appear as buckling modes.

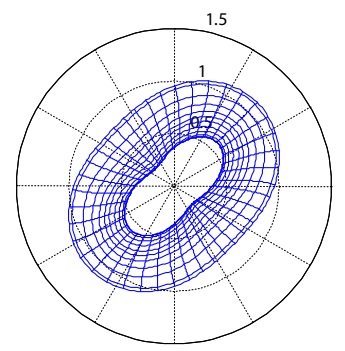

I

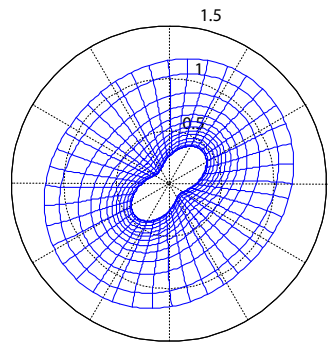

II

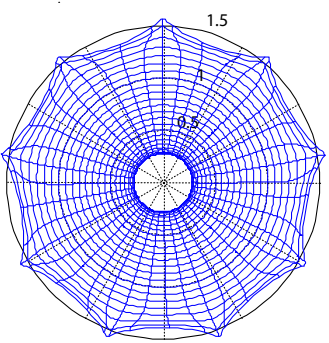

III

Fig. 3 Tube deformation after bifurcation corresponding to the points marked in Figure 2. Note that the amplitude (given by the choice of $\varepsilon$ has been chosen to illustrate the structure - it has no physical significance due to the nature of the linear stability analysis.

unstable and buckles, and the corresponding mode $n_{c r}$ is the critical buckling mode. For $P<P_{c r}^{*}$, the tube remains circular.

The value of $P_{c r}^{*}$ for $\gamma_{1}=1$ is the critical pressure without growth, which occurs for mode $n_{c r}=2$. Growth can have both stabilizing and destabilizing effects. For this thickness, radial resorption $\left(\gamma_{1}<1\right)$ is destabilizing: $P_{c r}^{*}$ is smaller for all values of $\gamma_{1}<1$ than for $\gamma_{1}=1$. On the other hand, radial growth $\left(\gamma_{1}>1\right)$ has a stabilizing effect initially but is destabilizing for large $\gamma_{1}$. Observe that the "strongest" tube possible, i.e. maximal value of $P_{c r}^{*}$, is for $\gamma_{1} \approx 1.72$, the point marked II in Figure 2. Notice also the bifurcation in critical 


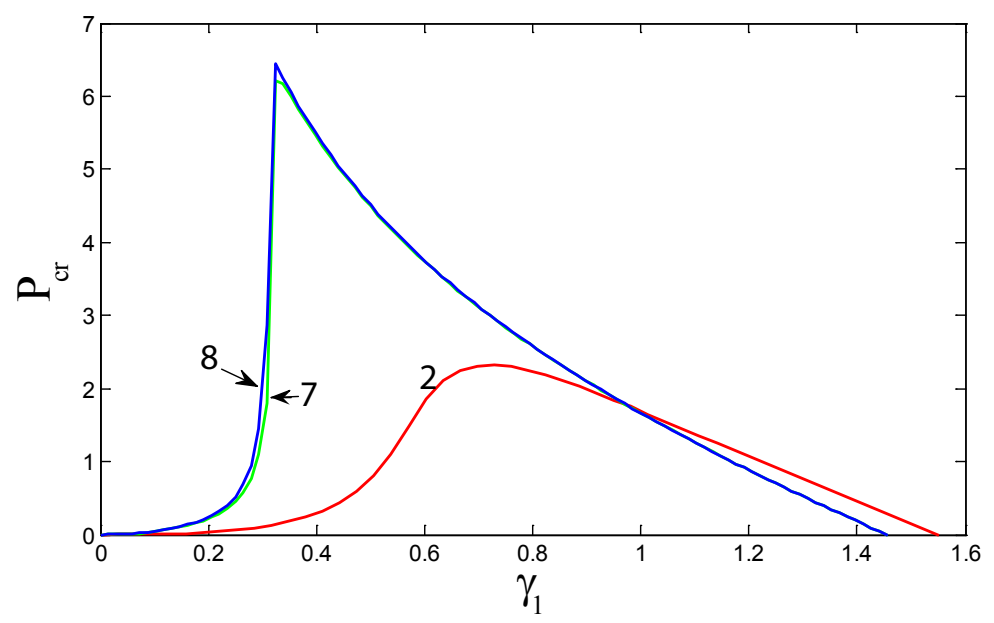

Fig. 4 Critical buckling pressure as a function of radial growth $\gamma_{1}$ for a thick tube, $A / B=0.1$. At this thickness, 3 modes are excitable. Modes 7 and 8 are almost identical, but a crossover does occur in the relevant region. With no growth, $n_{c r}=7$, but as $\gamma_{1}$ is increased the mode 8 curve just crosses below mode 7 so that $n_{c r}=8$ when $P=0$.

buckling mode that occurs at $\gamma_{1} \approx 1.95$. Interestingly, as $\gamma_{1}$ crosses this value, the buckling mode jumps from $n_{c r}=2$ to $n_{c r}=9$.

Along with the change in critical pressure, growth has a dramatic effect on the shape after bifurcation as well. Samples of the tube after deformation are provided in Figure 3 for the three points marked I,II,III in Figure 2. Note that point III corresponds to buckling due solely to differential growth (since $P_{c r}^{*}=0$ ). It is interesting that with increased radial growth, the buckling deformation moves from being mostly focused on the inner edge to being focused almost entirely on the outer edge, so that at point III the inner edge remains nearly circular.

Altering the thickness $A / B$ has several consequences. An equivalent diagram for a thick shell, $A / B=0.1$, is given in Figure 4. Note that the peaks of the curves have shifted to lower values of $\gamma_{1}$. In this case, radial growth is always destabilizing, whereas circumferential growth is initially stabilizing but then destabilizing. Hence, at this thickness, the "strongest" tube is obtained by radial resorption. This is somewhat counterintuitive. It is generally the case that without growth, a thicker tube is more stable, i.e. the critical pressure increases monotonically with the thickness. Yet in this case the critical pressure is maximized by radial resorption, a growth which serves to decrease the thickness. This behavior can be understood in terms of residual stress. Radial resorption creates a tensile residual stress, which makes the tube more stable mechanically. This combined effect highlights the interplay between mechanical and geometrical effects. For a thick tube, the mechanical benefit of radial resorption outweighs the geometric detriment of decreased thickness. For a thin tube, the situation is the opposite. An interesting consequence of this transition based on tube thickness is that there is a thickness, found to be $A / B=0.26$, for which the "strongest" tube occurs 
when $\gamma_{1}=1$. At this thickness, the peak of the curve $n=2$ is at $\gamma_{1}=1$, and any growth serves to destabilize the tube.

As might be expected, growth can have a much greater stabilizing effect on a thin shell than a thick one. To quantify the stabilization effect, define $P_{r}$ as the ratio of the maximal $P_{c r}^{*}$ and the value of $P_{c r}^{*}$ when $\gamma_{1}=1$. Note that by this definition, $P_{r}=1$ when $A / B=0.26$, the smallest value it can take. The dramatic effect of thickness is clear when comparing $P_{r}$ for thick versus thin tubes: for $A / B=0.1, P_{r}=1.39$; for $A / B=0.5, P_{r}=2.06$; and for $A / B=0.9, P_{r}=312$ ! Note that for $A / B=0.9, P_{c r}^{*} \approx 0.37$, a relatively small value, but still over 300 times greater than the extremely small pressure needed to buckle a thin tube.

Also, it is interesting to note that buckling due to pressure alone occurs at mode 2 for almost all thicknesses, while buckling due to growth alone is always at higher modes (greater than 6) and never occurs at mode 2 .

\subsection{Buckling in a constrained tube}

We have shown that anisotropic growth can cause buckling in the absence of external loads. If the geometry is constrained so that the outer boundary is held fixed, then isotropic growth, or volumetric swelling, can also induce buckling. This is illustrated in Figure 5, which plots the critical growth $\gamma_{1}=\gamma_{2}=: \gamma_{c r}$ at which buckling is induced by isotropic growth. Note that in this constrained geometry, growth induces pressure as the material pushes against the rigid outer boundary. Whereas mode 2 was most common in the unconstrained buckling considered in the previous subsections, a fixed circular outer boundary favors higher modes. Though many modes are almost indistinguishable over a large range of thickness, a crossover does occur, so that all modes greater than or equal to 7 may incrementally be excited as $A / B$ is increased. Interestingly, if the setup is inverted so that the inner radius is held fixed and circular, no amount of isotropic growth can induce buckling, at any mode. The difference with the fixed outer radius is that there is also a fixed and finite amount of area. From Figure 5 we see that $\gamma_{c r}$ is always greater than one, so that the the buckling is due to a combination of induced pressure on the outer boundary and expansion into a finite area.

\subsection{Thin tube analysis}

In the case of a thin tube, an asymptotic analysis can be carried out and explicit formulas established. The approach is outlined in Appendix A. The result is that if the tube thickness is small, i.e. $\delta:=B-A$ is a small parameter, then the critical strain at the inner edge, $\alpha_{a}=a / A$, can be obtained in an asymptotic expansion for each mode $n$ as

$$
\alpha_{a}^{(n)}=\alpha_{0}+\alpha_{1} \delta+\alpha_{2} \delta^{2}+O\left(\delta^{3}\right)
$$

where the formulas for the $\alpha_{i}$ are provided in Appendix A. Once $\alpha_{a}$ is known for a given mode, the critical pressure necessary to excite that mode can be computed via (5). The validity of the asymptotic approach is demonstrated in Figure 6, where numerical and asymptotic curves of the critical strains are plotted against the thickness $A / B$ for several modes and for growth ratio $\gamma_{1} / \gamma_{2}=1.5$. The incremental boundary conditions of zero normal and shear stress were utilized. The first curve that is encountered moving downward from $\alpha_{a}=1$ represents the critical mode and critical buckling strain. In this thin shell regime, the critical mode is 2 . We see that the asymptotic curves match well; the critical strain mode 2 is well approximated even at the thickness $A / B=0.7$ 


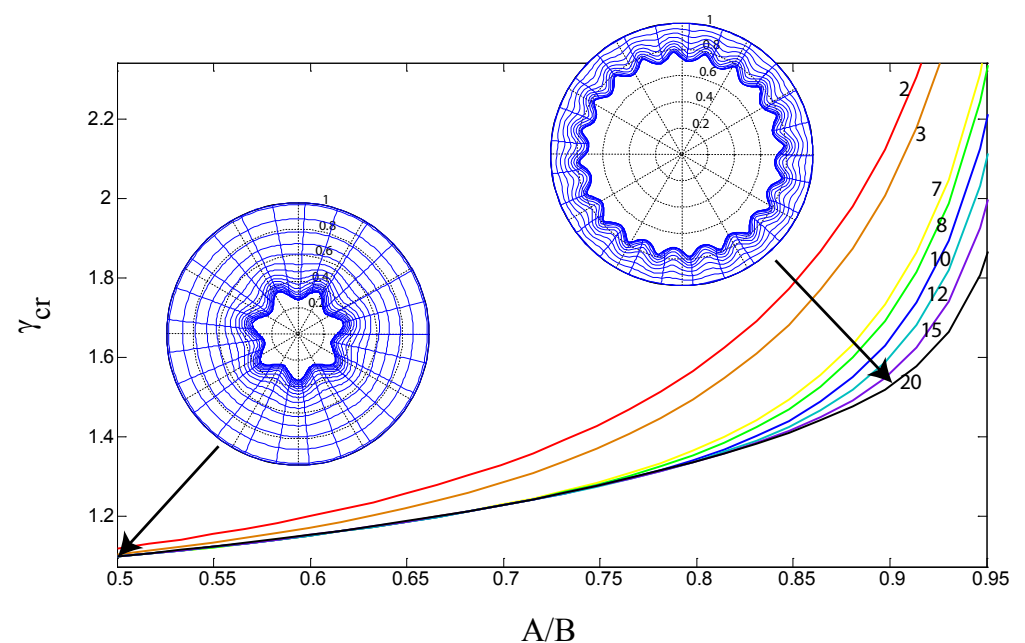

Fig. 5 Buckling induced by isotropic growth. Critical growth parameter $\gamma$ is plotted as a function of thickness for modes $n=2,3,7,8,10,12,15,20$. The form of the deformation appears for $A / B=0.5$ (mode 7 ) and $A / B=0.9$ (mode 20$)$.

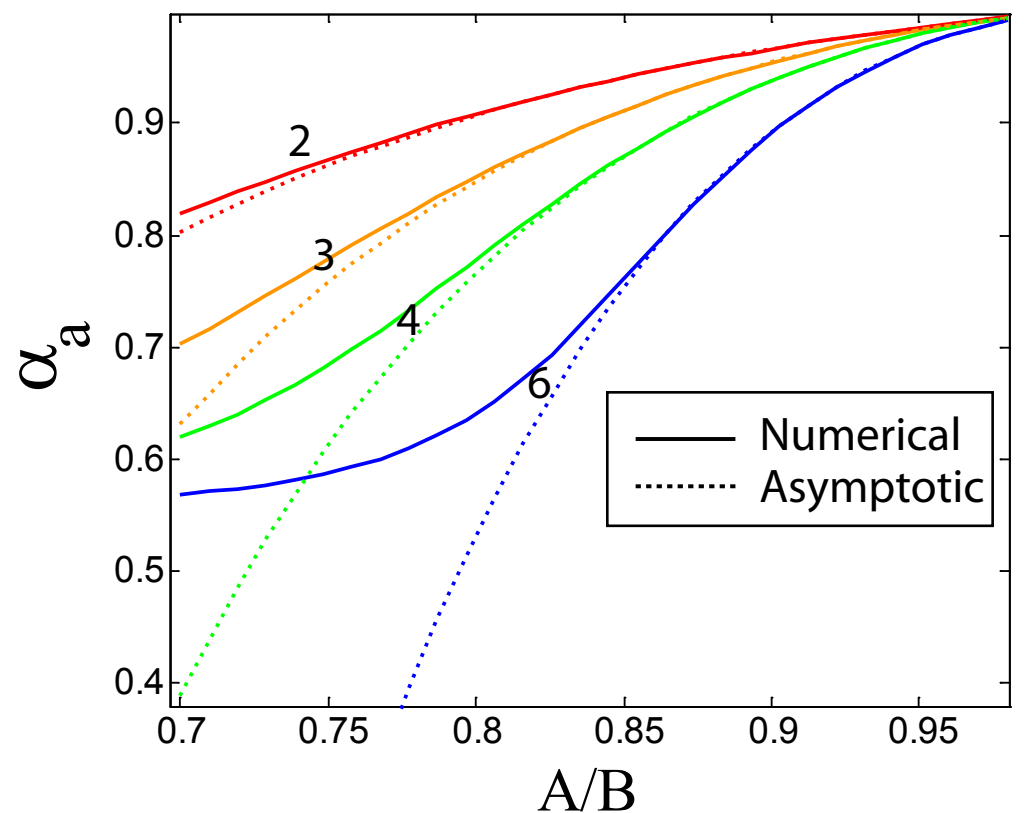

Fig. 6 Comparison of asymptotic (dotted) and numerical (solid) curves for the critical strain at the inner boundary, $\alpha_{a}$, versus thickness $A / B$ for modes $n=2,3,4,6 ; \gamma_{1} / \gamma_{2}=1.5$. 


\section{Two-layer model}

Many biological cylindrical structures are composed of two, or more, cylindrical layers bound together. Each layer may have different mechanical properties and be subject to anisotropic differential growth. Here we consider a two-layer tube. Rather than trying to fully explore the large parameter space, we focus on a range of parameter space relevant to biological applications, and demonstrate the effect of growth in this regime. Tissues such as the oesophagus or airways are comprised of a stiff inner layer, the mucosa, and a soft outer layer, the submucosa. Recalling that the inner region is described by $A \leq R \leq B$ and the outer region by $B \leq R \leq C$ in the initial configuration, in this section we will fix $A=1$, $C=1.5$, and either $B=1.1$ or $B=1.2$. We also fix the elastic moduli of the inner and outer layers to be $\mu_{1}=10, \mu_{2}=1$. Under this fixed geometry, we will investigate buckling as a function of the four growth parameters $\gamma_{1}^{(i)}, \gamma_{2}^{(i)}, \gamma_{1}^{(o)}$, and $\gamma_{2}^{(o)}$, the radial and circumferential growth in the inner and outer layers.

We first consider isotropic growth, but with different growth rates in each layer, and the incremental boundary conditions of zero normal and shear stress on the inner and outer edges. The critical pressure (smallest value over all modes) is plotted as a function of the ratio of growth rates $\gamma^{(i)} / \gamma^{(o)}$ in Figure 7 . The critical pressure only depends on the ratio; the deformation with different values of the growth parameters but with the same ratio is equivalent up to an isotropic and equal growth of both layers. Thus the point with $\gamma^{(i)} / \gamma^{(o)}=$ 1 is equivalent to the case of no growth. Hence, it is destabilizing for the inner layer to grow at a faster rate than the outer layer, and stabilizing for the inner layer to grow at a slower rate. This is somewhat interesting considering that the inner layer is much stiffer. Note also that the critical mode is $n_{c r}=2$ for every value of the growth ratio. The boundary conditions imply that both edges are unconstrained in the buckling deformation. This is not necessarily the case in biological tissues, however, where the outer edge is sometimes geometrically constrained. For instance, in the airways and oesophagus a third layer of smooth muscle surrounds the submucosa and enforces the outer edge to maintain a circular shape $[4,6]$. This geometrical constraint has a dramatic effect on the buckling. In Figure 8(a), the same graph is plotted as in Figure 7 but with the vanishing normal stress condition at $r=c$ replaced by the requirement that the perturbation vanish at the outer boundary. The critical mode is labeled at each point. There are several key differences that appear with this circular outer boundary condition. Most noticeably, the critical mode is never equal to 2, but rather increases incrementally from $n_{c r}=3$ to $n_{c r}=9$ as the growth ratio increases. Also, the buckling pressure is significantly higher, so that the tube is essentially stronger when the outer edge is kept circular. Buckling deformation shapes are included at the smallest and largest values of $\gamma^{(i)} / \gamma^{(o)}$ - notice that in the case $\gamma^{(i)} / \gamma^{(o)}=0.2$, the area inside the tube is much smaller, and the outer layer is proportionally much larger than the case $\gamma^{(i)} / \gamma^{(o)}=1.8$.

In Figure 8(b), the same graph is produced, but starting with a thicker initial inner layer, here $A=1, B=1.2, C=1.5$, and again $\mu_{1}=10, \mu_{2}=1$. As would be expected, increasing the thickness of the stiffer inner layer results in an overall increase in the buckling pressure. It also has the effect of reducing the buckling mode. The other interesting thing to note regards growth and stabilization. With the thicker inner layer, all isotropic growth serves to destabilize the tube, since the buckling pressure takes a minimum at the ratio $\gamma^{(i)} / \gamma^{(o)}=1$. Although it is difficult to see, the curve in Figure 8(a) takes a minimum value at a ratio greater than one. If the inner layer is further thickened, the minimum value occurs at a ratio less than one (not plotted). On the other hand, if the inner layer becomes thinner, the critical mode increases and the critical pressure decreases to the point where the minimum value of 


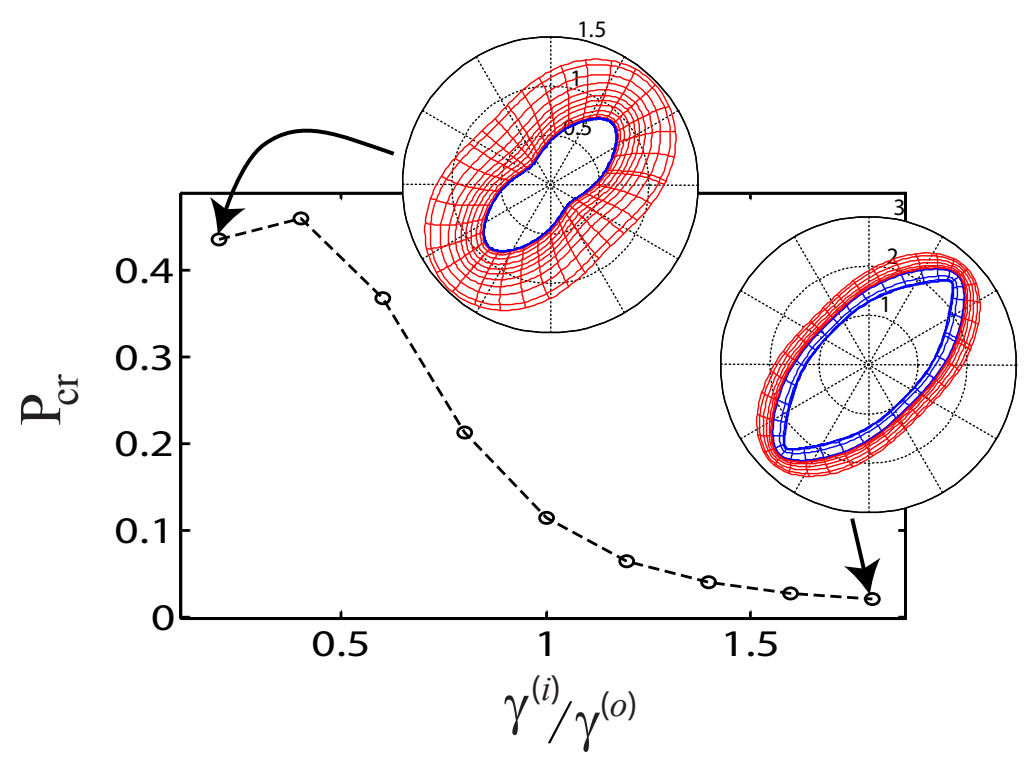

Fig. 7 Critical pressure as a function of growth rate ratio between inner and outer layers for isotropic growth in each layer. The incremental boundary conditions are zero traction on both surfaces. The critical mode is $n_{c r}=2$ at each point. Parameters are $A=1, B=1.1, C=1.5, \mu_{1}=10, \mu_{2}=1$.

the pressure curve touches the axis. At this thickness, there exists a growth ratio such that isotropic growth in each layer induces buckling without any external pressure.

As we have demonstrated in the case of a single layer, anisotropic growth can have a strong impact on the buckling properties of a tube. To briefly explore this effect in the case of a bilayer tube, we allow for anisotropic growth in the inner layer, and impose no growth in the outer layer. Again, only the ratio of radial to circumferential growth, $\gamma_{1}^{(i)} / \gamma_{2}^{(i)}$, factors into the buckling pressure. Figure 9 displays the critical pressure as a function of this ratio, ranging from 0.2 to 1.8 . A ratio greater than one corresponds to radial growth while a ratio less than one corresponds to circumferential growth or radial resorption of the inner layer. Radial growth has a two part effect: the inner layer becomes thicker, which for this thickness causes the tube to become more stable; at the same time the buckling mode decreases. Going the other direction, circumferential growth causes the inner layer to become thinner, the tube becomes less stable, and the buckling mode increases. The change in buckling mode is dramatic, from $n_{c r}=5$ at $\gamma_{1}^{(i)} / \gamma_{2}^{(i)}=1.8$ to $n_{c r}=28$ at $\gamma_{1}^{(i)} / \gamma_{2}^{(i)}=0.2$.

\subsection{Inverse problem}

The presence of four growth parameters in the two layer model has an interesting mathematical consequence that does not hold in the single layer model. For the two layer model, there are four equations that determine the bifurcation point and deformation: the two equations (17) for the finite deformation in each layer, the pressure condition (18) for the finite deformation, and the bifurcation ODE (13) for the incremental deformation. This suggests 


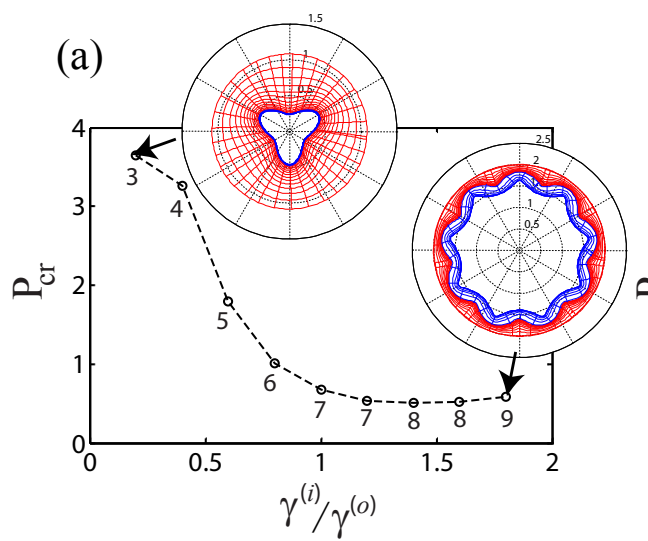

(b)

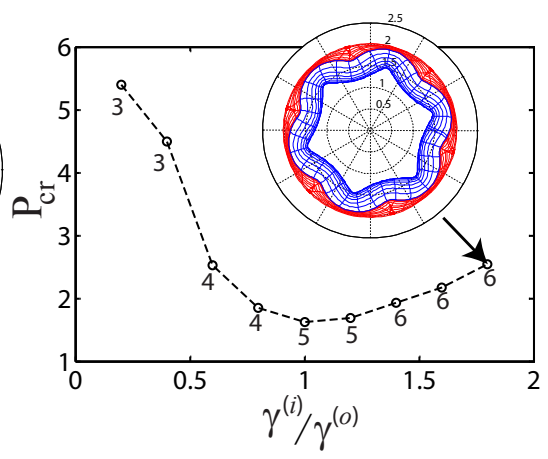

Fig. 8 Critical pressure as a function of growth rate ratio between inner and outer layers for isotropic growth in each layer, for a thinner inner layer $B=1.1$ (a) versus a thicker inner layer $B=1.2$ (b). In both cases, $A=1, C=1.5, \mu_{1}=10, \mu_{2}=1$. The circular outer boundary condition is used, so that the perturbation vanishes at the outer edge. The critical mode is labeled at each point, and select deformation patterns are included.

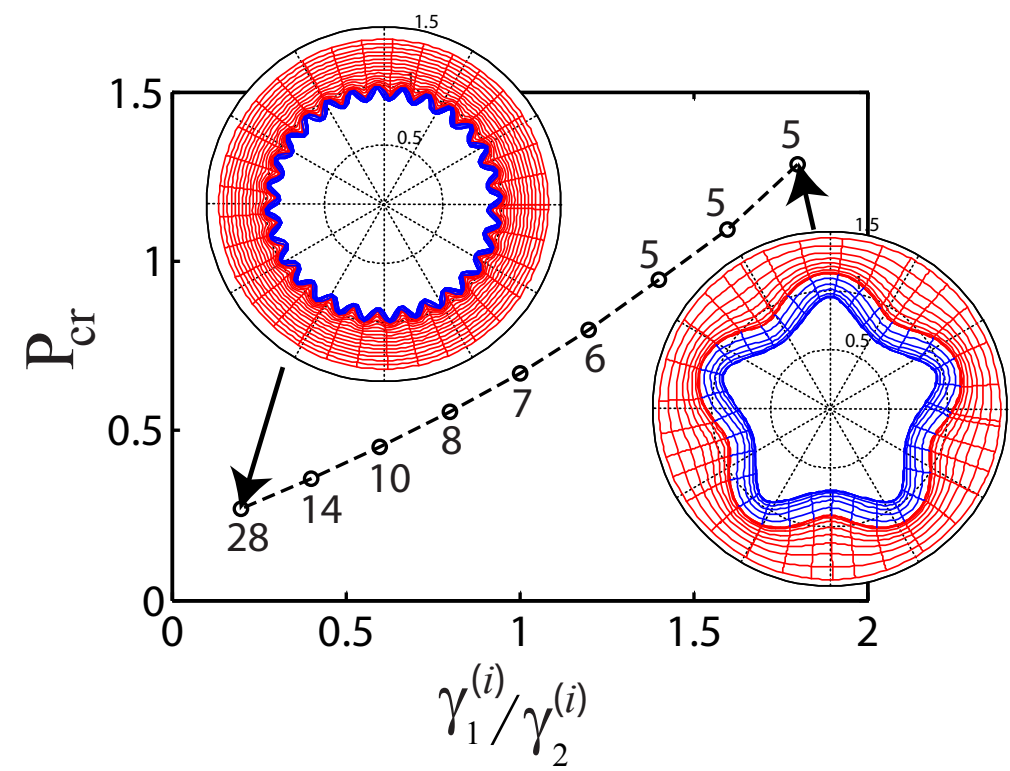

Fig. 9 Critical pressure as a function of ratio between radial and circumferential growth in inner layer, with no growth in outer layer. The circular outer boundary condition is used. The critical mode is labeled at each point, and select deformation patterns are included. $A=1, B=1.1, C=1.5, \mu_{1}=10, \mu_{2}=1$ 
that an inverse problem can be defined in which the bifurcation mode and critical pressure are given and the growth parameters which lead to that deformation are solved for. In other words, the typical "forward" approach we have used thus far is to take the growth parameters as input, use the pressure as a parameter in the bifurcation ODE, and find the critical value, from which the dimensions in the deformed state, i.e. $a, b, c$, can be determined. The "inverse" problem, on the other hand, is to take the values of $a, b, c$ and the critical pressure $P$ as input, use Equations (17) and (18) to relate the growth parameters, then the bifurcation ODE (13) can be written in terms of a single growth parameter. If a value is found that solves the bifurcation boundary value problem, then a form of growth is found that leads to the deformation specified by the input.

Note that this inverse problem cannot be formulated in a single layer model. With one layer, there are only two growth parameters but three equations describing the bifurcation and deformation: Equation (1) for the finite deformation, the pressure condition (5) for the finite deformation, and again the buckling ODE (13).

Due to the complexity of the equations, there is little hope for an analytical proof of the existence of a solution to the inverse problem. However, our simulations have suggested that a solution can be generally found. We demonstrate here with a rather dramatic example for the two layer model with $A=1, B=1.2, C=1.5, \mu_{1}=10, \mu_{2}=1$ and the circular outer boundary condition. Referring to Figure 9 , without growth the critical pressure is approximately $P_{c r} \approx 0.671$, the critical mode is 7 , and the dimensions at the point of buckling are $a=0.8794, b=0.9917, c=1.4225$. Suppose we seek a bifurcation such that the tube ends up significantly stronger and at the same time significantly thinner. We set $a=0.8794$, $b=0.9356$, and $c=1.1510$, so that both layers are half as thick at the point of buckling, and increase the critical pressure ten fold, to $P_{c r}=6.71$. We find that the inverse problem has the solution $\gamma_{1}^{(i)}=0.2266, \gamma_{2}^{(i)}=2.1410, \gamma_{1}^{(o)}=0.1410, \gamma_{2}^{(o)}=3.0650$.

An understanding of how growth is able to create a tube with the thickness of each layer cut in half and ten times more resistant to buckling can be gained by considering the stress fields and the growth without pressure. Figure 10 illustrates the idea. The initial configuration is shown at point I. Under the given growth, if no external pressure is applied the tube deforms to point II - a much larger radius with thinner layers. Since in each layer $\gamma_{2}>\gamma_{1}$, the growth is circumferential, which produces a tensile residual radial stress. The tensile stress serves to counteract the applied pressure, so that a large amount of pressure is required in deforming to the bifurcation point III. At the point of bifurcation, the radial stress is entirely transformed to a compressive stress.

It must be pointed out that the critical buckling mode cannot be selected with this inverse analysis. That is, for fixed values of $a, b, c$ and $P$ and $a$ chosen mode, growth parameters can be found such that that particular mode is excited at that deformation. For those growth parameters though, there is no guarantee that a different mode is not excited at lower pressure. Presumably, a third layer would give one more free growth parameter, and mathematically the critical mode could also be selected. We leave such an analysis, as well as analytical treatment of the inverse problem, for future work.

\section{Conclusion}

We have investigated the mechanical effect of growth and residual stress on the circumferential buckling of a cylindrical elastic tube. Material and geometric nonlinearities as well as differential growth were taken into account by formulating the problem within the theory of morphoelasticity. We studied the role of growth in buckling in a broad mechanical sense and 


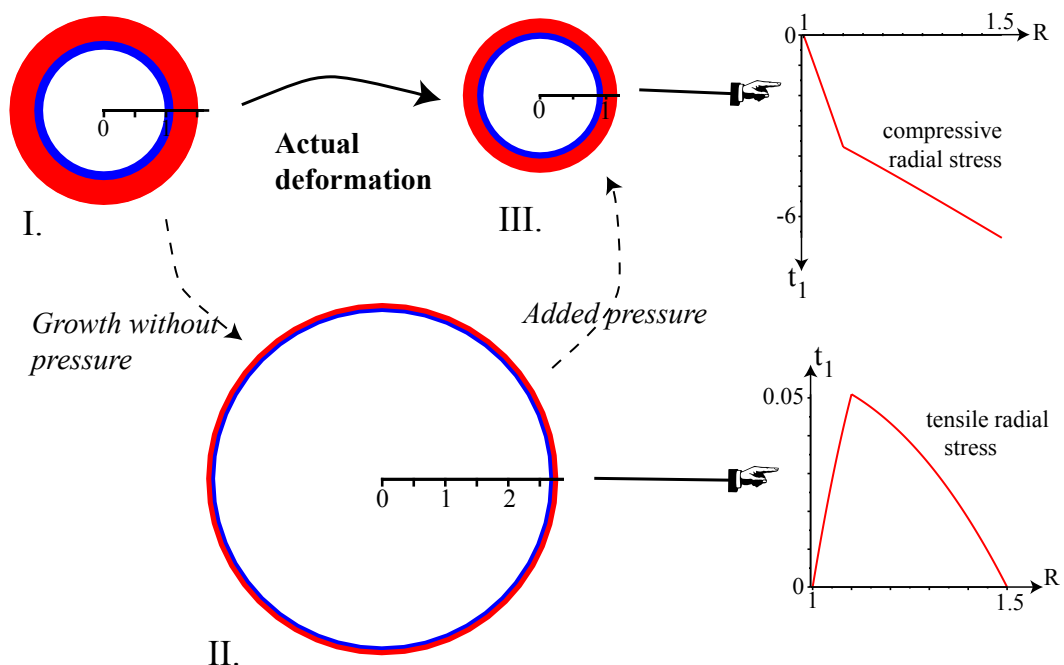

Fig. 10 Schematic of how differential growth can lead to a tube with thinner layers but that is ten times stronger, i.e. buckles at a pressure greater by a factor of 10 than the buckling pressure with no growth. The growth without pressure produces a tensile residual stress, which opposes the external pressure. The plots of the anular regions are to scale and correspond to the parameter values in the text.

have shown that growth can have a substantial impact on the buckling pressure and mode, and in fact that growth alone can induce buckling, either through residual stress generated by anisotropic growth, through isotropic growth in a constrained geometry, or by isotropic growth with different growth rates in a bilayer tube.

The model presented here represents a simplified and idealized structure, assuming axial uniformity and the buckling of circular cross sections to non-circular shapes with $n$ symmetry axes. A potentially important drawback of the incremental theory to study buckling is that no information can be extracted about the deformation beyond the point of bifurcation - only the critical pressure and mode can be found, information about the size of the perturbation or any further deformation require a different approach. Nevertheless, our analysis demonstrates the initimate relationship between anisotropic growth rates, the buckling properties of pressure and mode, and constrained versus unconstrained outer boundaries. In particular, we have shown that stability depends on both mechanical and geometrical factors, so that in determining the strength of a thicker versus a thinner tube, for instance, residual stress must also be considered. The effect of residaul stress has potentially profound consequences, in particular in the case of a two-layer tube, where four growth parameters enables for the design of a tube with chosen bifurcation properties.

Acknowledgments: This publication is based on work supported by Award No. KUK-C1013-04, made by King Abdullah University of Science and Technology (KAUST), and based in part upon work supported by the National Science Foundation under grant DMS0907773 (AG) 
Appendix A: Thin shell analysis

Here we outline the asymptotic analysis in the case of a thin shell. The idea, implemented previously for spherical shells [30,18], is to introduce the small parameter $\varepsilon:=b-a$ and the stretched variable

$$
x=\frac{r-a}{\varepsilon} .
$$

Letting $u(x)=f(r)$, we can recast the bifurcation ODE (13) and the boundary conditions (14) in terms of $x$ and $u(x)$. The same as in the numerical procedure, we treat the boundary value problem as an initial value problem and solve two copies of the system, but here the two copies are solved in an asymptotic expansion in $\varepsilon$. That is, we let $u(x)=b_{1} \phi_{1}(x)+$ $b_{2} \phi_{2}(x)$, where each

$$
\phi_{i}=\sum_{j=0}^{N} \varepsilon^{j} \phi_{i j}
$$

Making these substitutions and expanding the system in $\varepsilon$, the $\phi_{i j}(x)$ can be solved exactly, and the initial conditions at $x=0$ are chosen so that the vectors $\left[\phi_{1}(0), \phi_{1}^{\prime}(0), \phi_{1}^{\prime \prime}(0), \phi_{1}^{\prime \prime \prime}(0)\right]$, $\left[\phi_{2}(0), \phi_{2}^{\prime}(0), \phi_{2}^{\prime \prime}(0), \phi_{2}^{\prime \prime \prime}(0)\right]$ are linearly independent.

Once the $\phi_{i j}$ are known, the determinant of boundary conditions at $x=1$ is formed,

$$
D(a ; \varepsilon)=\left|\begin{array}{ll}
c_{1}\left(\phi_{1}(1)\right) & c_{1}\left(\phi_{2}(1)\right) \\
c_{2}\left(\phi_{1}(1)\right) & c_{2}\left(\phi_{2}(1)\right)
\end{array}\right|
$$

Writing $a=a_{0}+\varepsilon a_{1}+\varepsilon a^{2}+\ldots$ and expanding $D(a ; \varepsilon)=0$ in powers of $\varepsilon$ enables us to solve for $a_{i}$ at each order. Dividing by $A$ to put the formula in terms of the critical strains, the solution $\alpha_{a}=a / A$ reads to third order

$$
\alpha_{a}=\alpha_{0}+\tilde{\alpha_{1}} \varepsilon+\tilde{\alpha_{2}} \varepsilon^{2}+O\left(\varepsilon^{3}\right)
$$

where $\alpha_{0}$ solves

$$
\begin{gathered}
\alpha_{0}^{8}+2 \gamma_{2}^{4} \alpha_{0}^{4}-3 \gamma_{2}^{8}=0, \\
\tilde{\alpha_{1}}=-\frac{3 \gamma_{2}^{9} \gamma_{1}-2 \alpha_{0}^{6} \gamma_{2}^{4}-3 \gamma_{2}^{9} A \alpha_{0} \gamma_{1}+2 \alpha_{0}^{5} A \gamma_{2}^{5} \gamma_{1}+\alpha_{0}^{9} A \gamma_{2} \gamma_{1}-2 \alpha_{0}^{10}+\alpha_{0}^{8} \gamma_{2} \gamma_{1}}{2 A \gamma_{2} \gamma_{1}\left(3 \alpha_{0}^{8}+4 \alpha_{0}^{4} \gamma_{2}^{4}-3 \gamma_{2}^{8}\right)},
\end{gathered}
$$


and

$$
\begin{aligned}
\tilde{\alpha_{2}}= & {\left[816 \alpha_{0}^{22} \gamma_{2}^{7} \gamma_{1}+1284 \alpha_{0}^{18} \gamma_{2}^{11} \gamma_{1}+625 \gamma_{2}^{20} \gamma_{1}^{2} \alpha_{0}^{8}-864 \alpha_{0}^{7} A \gamma_{2}^{23} \gamma_{1}-1356 \alpha_{0}^{19} A \gamma_{2}^{11} \gamma_{1}\right.} \\
& -1440 \alpha_{0}^{23} A \gamma_{2}^{7} \gamma_{1}-1696 \alpha_{0}^{14} \gamma_{2}^{15} \gamma_{1}+780 \alpha_{0}^{10} \gamma_{2}^{19} \gamma_{1}-144 \alpha_{0}^{6} \gamma_{2}^{23} \gamma_{1}+2304 \alpha_{0}^{15} A \gamma_{2}^{15} \gamma_{1} \\
& +396 \alpha_{0}^{11} A \gamma_{2}^{19} \gamma_{1}-36 n^{2} \gamma_{2}^{28} \gamma_{1}^{2}-18 n^{2} \alpha_{0}^{28} \gamma_{1}^{2}-918 \alpha_{0}^{20} \gamma_{2}^{8} \gamma_{1}^{2}-666 \alpha_{0}^{10} A^{2} \gamma_{2}^{20} \gamma_{1}^{2} \\
& +528 \alpha_{0}^{14} A^{2} \gamma_{2}^{16} \gamma_{1}^{2}+234 \alpha_{0}^{18} A^{2} \gamma_{2}^{12} \gamma_{1}^{2}+48 \alpha_{0}^{22} A^{2} \gamma_{2}^{8} \gamma_{1}^{2}+876 \alpha_{0}^{21} A \gamma_{2}^{8} \gamma_{1}^{2}+63 \alpha_{0}^{24} \gamma_{2}^{4} \gamma_{1}^{2} \\
& +36 \alpha_{0}^{26} \gamma_{2}^{3} \gamma_{1}-252 \alpha_{0}^{27} A \gamma_{2}^{3} \gamma_{1}+54 \alpha_{0}^{25} A \gamma_{2}^{4} \gamma_{1}^{2}+972 \gamma_{2}^{27} \alpha_{0}^{2} \gamma_{1}+18 \alpha_{0}^{26} A^{2} \gamma_{2}^{4} \gamma_{1}^{2} \\
& -324 \gamma_{2}^{27} \alpha_{0}^{3} A \gamma_{1}-162 \gamma_{2}^{28} A^{2} \alpha_{0}^{2} \gamma_{1}^{2}+956 \alpha_{0}^{20} \gamma_{2}^{10}+2272 \alpha_{0}^{16} \gamma_{2}^{14}-924 \alpha_{0}^{12} \gamma_{2}^{18} \\
& -72 \alpha_{0}^{24} \gamma_{2}^{6}-1368 \alpha_{0}^{8} \gamma_{2}^{22}+324 \alpha_{0}^{4} \gamma_{2}^{26}-36 \alpha_{0}^{28} \gamma_{2}^{2}-1881 \gamma_{2}^{28} \gamma_{1}^{2}-192 n^{2} \alpha_{0}^{24} \gamma_{2}^{4} \gamma_{1}^{2} \\
& -614 n^{2} \alpha_{0}^{20} \gamma_{2}^{8} \gamma_{1}^{2}-580 n^{2} \alpha_{0}^{16} \gamma_{2}^{12} \gamma_{1}^{2}+322 n^{2} \alpha_{0}^{12} \gamma_{2}^{16} \gamma_{1}^{2}+488 n^{2} \alpha_{0}^{8} \gamma_{2}^{20} \gamma_{1}^{2}-138 n^{2} \alpha_{0}^{4} \gamma_{2}^{24} \gamma_{1}^{2} \\
& +2586 \gamma_{2}^{24} \gamma_{1}^{2} \alpha_{0}^{4}+666 \gamma_{2}^{20} A \gamma_{1}^{2} \alpha_{0}^{9}-756 \gamma_{2}^{24} A \gamma_{1}^{2} \alpha_{0}^{5}+1134 \gamma_{2}^{28} A \gamma_{1}^{2} \alpha_{0}-696 \gamma_{2}^{16} A \gamma_{1}^{2} \alpha_{0}^{13} \\
& \left.+1794 \gamma_{2}^{12} A \gamma_{1}^{2} \alpha_{0}^{17}-3831 \gamma_{2}^{12} \gamma_{1}^{2} \alpha_{0}^{16}-2148 \gamma_{2}^{16} \gamma_{1}^{2} \alpha_{0}^{12}\right] \\
& \times\left[48 A^{2} \alpha_{0} \gamma_{2}^{4} \gamma_{1}^{2}\left(3 \alpha_{0}^{8}+4 \alpha_{0}^{4} \gamma_{2}^{4}-3 \gamma_{2}^{8}\right)^{3}\right]^{-1}
\end{aligned}
$$

In terms of the reference variables, we define $\delta:=B-A$. Then, from the relationship (1) we have $b^{2}-a^{2}=\gamma_{1} \gamma_{2}\left(B^{2}-A^{2}\right)$, which expanded to second order reads

$$
\varepsilon=\frac{\gamma_{1} \gamma_{2}}{\alpha_{0}} \delta
$$

and the critical strain can be written as

$$
\alpha_{a}=\alpha_{0}+\alpha_{1} \delta+\alpha_{2} \delta^{2}+O\left(\delta^{3}\right)
$$

where $\alpha_{i}=\left(\frac{\gamma_{1} \gamma_{2}}{\alpha_{0}}\right)^{i} \tilde{\alpha}_{i}$

\section{References}

1. L. Euler. Sur la force des colonnes. Mem. Acad., Berlin, 1759.

2. A. Goriely, R. Vandiver, and M. Destrade. Nonlinear Euler buckling. Proceedings of the Royal Society A: Mathematical, Physical and Engineering Sciences, 464(2099):3003-3019, 2008.

3. J. P. Renaudeaux and B. Dion. Dispositif d'injection de médicaments en phase liquide. Brevet, 91:01790, 1991.

4. R. K. Lambert, S. L. Codd, M. R. Alley, and R. J. Pack. Physical determinants of bronchial mucosal folding. J. Appl. Physiol., 77:1206-1216, 1994.

5. D. Liao, J. Zhao, J. Yang, and H. Gregersen. The oesophageal zero-stress state and mucosal folding from a giome perspective. World J. Gastroenterol, 13(9):1347-1351, 2007.

6. W. Yang, T. C. Fung, K. S. Chian, and C. K. Chong. Instability of the two-layered thick-walled esophageal model under the external pressure and circular outer boundary condition. J. Biomech., 40:481-490, 2007

7. M. M. L. Lee and S. Chien. Morphologic effects of pressure changes on canine carotid artery endothelium as observed by scanning electron microscopy. The Anatom. Rec., 194:1-14, 1978.

8. X. Lu, J. Zhao, and H. Gregersen. Small intestinal morphometric and biomechanical changes during physiological growth in rats. J. Biomech., 38:417-426, 2005.

9. I. Tadjbakhsh and F. Odeh. Equilibrium states of elastic rings. J. Math. Anal. Appl., 18:59-74, 1967.

10. R. H. Moreno, J. C. Hogg, and P. D. Paré. Mechanics of airway narrowing. Am. Rev. Respir. Dis., 133:1171-1180, 1986. 
11. R. K. Lambert. Role of bronchial basement membrane in airway collapse. J. Appl. Physiol., 71:666-673, 1991.

12. B. Dion, S. Naili, J. P. Renaudeaux, and C. Ribreau. Buckling of elastic tubes: study of highly compliant device. Med. \& Biol. Eng. \& Comp., 33(2):196-201, 1995.

13. B. R. Wiggs, C. A. Hrousis, J. M. Drazen, and R. D. Kamm. On the mechanism of mucosal folding in normal and asthmatic airways. J. Appl. Physiol, 83:1814-1821, 1997.

14. C. A. Hrousis, B. R. Wiggs, J. M. Drazen, D. M. Parks, and R. D. Kamm. Mucosal folding in biologic vessels. J. Biomech. Eng., 124(4):334-341, 2002.

15. H. L. Huber and K. K. Koessler. The pathology of bronchial asthma. Arch. Intern. Med., 30:689-760, 1922.

16. A. L. James, P. D. Paré, and J. C. Hogg. The mechanics of airway narrowing in asthma. Am. Rev. Respir. Dis., 139(1):242-246, 1989.

17. A. E. Redington and P. H. Howarth. Airway wall remodelling in asthma. Thorax, 52:310-312, 1997.

18. M. B. Amar and A. Goriely. Growth and instability in elastic tissues. J. Mech. Phys. Solids, 53:22842319, 2005.

19. R. M. Vandiver. Morphoelasticity: the mechanics and mathematics of elastic growth. PhD thesis, U. of Arizona, 2009

20. Y. C. Fung. What are the residual stresses doing in our blood vessels? Annals Biomed. Eng., 19:237-249, 1991.

21. J. D. Humphrey. Continuum biomechanics of soft biological tissues. Proc. Roy. Soc. Lond. A, 459:3-46, 2003.

22. R. Vandiver and A. Goriely. Tissue tension and axial growth of cylindrical structures in plants and elastic tissues. Europhys. Lett., 84:58004, 2008.

23. D. E. Moulton and A. Goriely. Possible role of differential growth in airway wall remodeling in asthma. J. Appl. Physiol. preprint.

24. E. K. Rodriguez, A. Hoger, and A. McCulloch. Stress-dependent finite growth in soft elastic tissues. J. Biomech, 27:455-467, 1994.

25. A. Goriely and M. Ben Amar. Differential growth and instability in elastic shells. Phys. Rev. Lett., 94(19):198103, 2005.

26. R. Vandiver and A. Goriely. Morpho-elasto-dynamics: The long-time dynamics of elastic growth. International Journal of Biological Dynamics, In press, 2008.

27. A. Goriely and D. E. Moulton. New Trends in the Physics and Mechanics of Biological Systems, chapter Morphoelasticity - A theory of elastic growth (M. Ben Amar, A. Goriely, M. Mueller, Editors) Oxford University Press, 2010.

28. A. Goriely and R. Vandiver. On the mechanical stability of growing arteries. IMA J. Appl. Math., Accepted:122, 2010.

29. R. W. Ogden. Non-linear Elastic Deformation. Dover, New York, 1984.

30. Y. Fu. Some asymptotic results concerning the buckling of a spherical shell of arbitrary thickness. Int. J. Nonlinear Mech., 33:1111-1122, 1998. 



\section{RECENT REPORTS}

13/10 Nonlinear Morphoelastic Plates II: Exodus to Buckled States

McMahon

Goriely

Tabor

14/10 Analysis of Brownian dynamics simulations of reversible biomolecular reactions

Lipkova

Zygalakis

Chapman

Erban

15/10 Travelling waves in hyperbolic chemotaxis equations

Xue

Hwang

Painter

Erban

16/10 The Physics and Mechanics of Biological Systems

Goriely

Moulton

17/10 Crust formation in drying colloidal suspensions

Style

Peppin

18/10 A Mathematical Model of Tumor-Immune Interactions

Robertson-Tessi

El-Kareh

Goriely

19/10 Elastic cavitation, tube hollowing, and differential growth in plants and biological tissues

Goriely

Moulton

Vandiver

20/10 Asymptotic expressions for the nearest and furthest dislocations

Hall in a pile-up against a grain boundary

21/10 Cardiac electromechanics: the effect of contraction model on the mathematical problem and accuracy of the numerical scheme

Pathmanathan

Chapman

Gavaghan

Whiteley

22/10 Fat vs. thin threading approach on GPUs: application to stochastic simulation of chemical reactions

Klingbeil

Erban

Giles

Maini

23/10 Asymptotic analysis of a system of algebraic equations arising in dislocation theory

Hall

Chapman

Ockendon

25/10 Preconditioning for Allen-Cahn Variational Inequalities with NonLocal Constraints

Blank

Sarbu

Stoll

26/10 On an evolution equation for sand dunes

Ellis

Fowler

27/10 On Liquid Films on an Inclined Plate

Benilov

Chapman

McLoed

Ockendon

Zubkov

28/10 An a posteriori error analysis of a mixed finite element Galerkin 
30/10 The Landau-de Gennes theory of nematic liquid crystals: Uniax- Majumdar iality versus Biaxiality

31/10 The Radial-Hedgehog Solution in Landau-de Gennes' theory Majumdar

32/10 Nonlinear instability in flagellar dynamics: a novel modulation Gadelha mechanism in sperm migration?

Gaffney

Smith

Kirkman-Brown

33/10 Error bounds on block GaussSeidel solutions of coupled multiphysics problem

Whiteley

Gillow

Tavener

Walter

34/10 A random projection method for sharp phase boundaries in lattice

Reis Boltzmann simulations

Dellar

35/10 Regularized Particle Filter with Langevin Resampling Step Duan

Farmer

Moroz

35/10 Sequential Inverse Problems Bayesian Principles and the Logistic

Duan Map Examplep

Farmer

Moroz

Copies of these, and any other OCCAM reports can be obtained from:

Oxford Centre for Collaborative Applied Mathematics

Mathematical Institute

24 - 29 St Giles'

Oxford

OX1 3LB

England

www.maths.ox.ac.uk/occam 